EDUCATION

Research, Innovation and Solutions on-line ${ }^{(0)}$
Electronic Journal of Research

in Educational Psychology

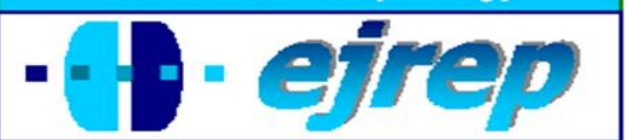

\title{
Aportaciones de la Psicología de la Innovación y del Emprendimiento a la Educación, en la Sociedad del Conocimiento
}

\author{
Jesús de la Fuente Arias ${ }^{1}$, \\ Manuel Mariano Vera Martínez ${ }^{2}$ \\ Maria Cardelle-Elawar ${ }^{3}$
}
${ }^{1}$ Dpto. Psicología Evolutiva y de la Educación, Universidad de Almería
${ }^{2}$ Centro Universitario de Magisterio "La Inmaculada", Universidad de Granada ${ }^{3}$ Arizona State University. USA

\section{España / EEUU}

Correspondencia: Jesús de la Fuente Arias. Education \& Psychology I+D+i. C/ Costa Azul, 68. 04009 Almería, SPAIN. E-mail: jfuente@ual.es 


\section{Resumen}

En la actualidad, la Sociedad Globalizada del Conocimiento del siglo XXI, ha supuesto grandes cambios en los modelos de trabajo y en nuestras propias vidas, asociados a la revolución de las Tecnologías de la Información y de la Comunicación (TICs), lo que ha llevado consigo nuevas maneras de entender el propio conocimiento, las actividades humanas y, por ende, las actividades profesionales y económicas. En este contexto actual, socioeducativo y socioeconómico, parece que tiene sentido -más que nunca- plantearse si deben producirse ajustes en la misión y visión de los modelos educativos al uso, es decir, en las finalidades educativas que se pretenden, para reajustar las mismas a la luz de las nuevas demandas contextuales de formación en las generaciones del siglo XXI.

Este trabajo se estructura, en varias partes. En primer lugar, se definen los conceptos Creatividad, Innovación y Emprendimiento, así como sus relaciones y papel en la cadena de valor I+D+i. A continuación se justifica por qué la Sociedad del Conocimiento necesita personas con creatividad, innovación y emprendimiento. En el tercer punto, nuclear de esta revisión, se aborda la necesidad y aportaciones de la Psicología de la Innovación y del Emprendimiento a los procesos educativos centrados en el desarrollo de estas competencias. En cuarto lugar, se proponen las implicaciones de las aportaciones anteriores en un nuevo programa educativo emergente, la Educación para la Competencia en Innovación y Emprendimiento, analizando los aprendizajes propios de esta competencia, así como las propuestas curriculares a integrar transversalmente en los procesos educativos. Se finaliza con las conclusiones y las implicaciones educativas de esta propuesta.

Palabras clave: Psicología de la innovación, Psicología del Emprendimiento, Educación para la Innovación y el Emprendimiento, Competencia en innovación y Emprendimiento, Sociedad del Conocimiento. 


\begin{abstract}
The globalized Knowledge Society of the $21^{\text {st }}$ century brings with it important changes in models of work and lifestyle, triggered by the revolution in Information and Communication Technologies (ICTs). It has led to new ways of understanding knowledge itself, human activity, and consequently, professional and economic activity. In this current socio-educational and socio-economic context, more than ever, it makes sense to ask whether the mission and vision of commonly used educational models, that is, the educational purposes pursued, should be adjusted in the light of new context-driven training demands for the present and upcoming generations.
\end{abstract}

This paper is structured in several parts. First, the concepts of Creativity, Innovation and Entrepreneurship are defined, as well as their relationships and role in the R\&D\&I value chain. Next is a justification of why the Knowledge Society needs persons with creativity, innovation and entrepreneurship. Third, and at the heart of this review, we address the need for a Psychology of Innovation and Entrepreneurship and its contributions to educational processes for developing these competencies. Fourth, we suggest the implications of such contributions in an emerging educational paradigm, Education for Competency in Innovation and Entrepreneurship, where we analyze what kind of learning would be involved in this competency, as well as cross-curriculum proposals to be integrated across educational processes. Conclusions and further educational implications are discussed in closing.

Key words: Psychology of Innovation, Psychology of Entrepreneurship, Education for Innovation and Entrepreneurship, Competency in Innovation and Entrepreneurship, Knowledge Society.

Received: 10/02/12 Initial acceptance: 10/10/12 Final acceptance: 11/23/12 


\section{Introducción}

Uno de los retos permanentes de cualquier sistema educativo (formal, no formal o informal), es promover el desarrollo integral y las competencias de las nuevas generaciones, para su feliz y plena integración en el contexto sociocultural y económico que les ha tocado vivir. En la medida en que el propio sistema contribuya a ello, estará dando respuesta a su misión; si, por el contrario, esto no se realiza, se estará perdiendo la oportunidad de dotar a los nuevos ciudadanos de herramientas instrumentales para su inserción laboral y su plena integración en las sociedades complejas del siglo XXI.

Para conocer las finalidades educativas que debe tener un sistema dado, y si las mismas están bien diseñadas, basta realizar un análisis pormenorizado del contexto socioeducativo y socioeconómico, en el que van a vivir los sujetos, objeto de tales finalidades. En la actualidad, la Sociedad Globalizada del Conocimiento del siglo XXI, ha supuesto grandes cambios en los modelos de trabajo y en nuestras propias vidas, asociados a la revolución de las Tecnologías de la Información y de la Comunicación (TICs), lo que ha llevado consigo nuevas maneras de entender el propio conocimiento, las actividades humanas y, por ende, las actividades profesionales y económicas. Además, la interconexión global planetaria ha supuesto un nuevo modelo de negocios, basado en las TICs, y rápidos procesos de cambio e innovación. Paralelamente, la crisis de los modelos de negocio y de profesionalización tradicionales - basados en la cercanía al hogar y en la reproducción de los roles profesionales paternos- en aras de la globalización, la innovación en los nichos de mercado y la diversificación, ha supuesto un antes y un después, en la forma de entender la capacitación y las vías de acceso al mercado laboral.

En este contexto actual socioeducativo y socioeconómico, parece que tiene sentido más que nunca- plantearse si deben producirse ajustes en la misión y visión de los modelos educativos al uso, es decir, en las finalidades educativas que se pretenden, para reajustar las mismas a la luz de las nuevas demandas contextuales de formación en las generaciones del siglo XXI. Ante tal reto, la incipiente Psicología de la Innovación y del Emprendimiento -que en este trabajo se postula- puede aportar elementos importantes de análisis respecto a la situación educativa actual, a la par que tiene capacidad para proponer directrices e implicaciones educativas, basadas en la investigación y en el espíritu empírico que le caracteriza. También puede realizar aportaciones relevantes, para integrar la innovación, el emprendimiento y en la 
cadena de valor $\mathrm{I}+\mathrm{D}+\mathrm{i}$, en los procesos educativos actuales, si deseamos que los futuros ciudadanos comprendan el valor de la misma en la prosperidad y en la economía basada en el conocimiento.

Para tal fin, este trabajo se estructura, en varias partes. En primer lugar, se definen los conceptos Creatividad, Innovación y Emprendimiento, así como sus relaciones y papel en la cadena de valor I+D+i. A continuación, se justifica por qué la Sociedad del Conocimiento necesita personas con creatividad, innovación y emprendimiento. En el tercer punto, nuclear de esta revisión, se aborda la necesidad y aportaciones de la Psicología de la Innovación y del Emprendimiento a los procesos educativos centrados en el desarrollo de estas competencias. En cuarto lugar, se proponen las implicaciones de las aportaciones anteriores en un nuevo paradigma educativo emergente, la Educación para la Competencia en Innovación y Emprendimiento, analizando los aprendizajes propios de esta competencia, así como las propuestas curriculares a integrar transversalmente en los procesos educativos. Se finaliza con las conclusiones y las implicaciones educativas de esta propuesta.

\section{Creatividad, Innovación y Emprendimiento}

¿Qué son la innovación y el emprendimiento? ¿Qué relación tienen con la creatividad? Según el Diccionario de la Real Académia Española, la creatividad se refiere a engendrar. Este es el significado literal que tiene el término creatividad y se obtiene al establecer el origen etimológico de aquel. La creatividad es el proceso de presentar un problema a la mente con claridad (ya sea imaginándolo, visualizándolo, suponiéndolo, meditando, contemplando, etc.) y luego originar o inventar una idea, concepto, noción o esquema, según líneas nuevas o no convencionales. Supone estudio y reflexión más que acción.

Creatividad es la capacidad de ver nuevas posibilidades y hacer algo al respecto. Cuando una persona va más allá del análisis de un problema e intenta poner en práctica una solución se produce un cambio. A esto se llama creatividad: ver un problema, tener una idea, hacer algo sobre ella, tener resultados positivos. Los miembros de una organización tienen que fomentar un proceso que incluya oportunidades para el uso de la imaginación, la experimentación y acción. La sinéctica es una disciplina que desarrolla métodos o conjuntos de estrategias cuyo propósito es desarrollar la creatividad y la productividad. 
La Innovación, es definida por el diccionario de la Real Academia, como cualquier cambio que introduce alguna novedad o varias, o también, como sinónimo de creación. Es la capacidad de generar e implementar una nueva idea, producto, servicio u negocio con éxito en el mercado, de obtener un beneficio tangible a partir de la generación de ideas, de identificar oportunidades y para el aprovechamiento exitoso de estas. En definitiva, la capacidad de convertir ideas en valor (Reyes, 2009). Sin embargo, el emprendimiento, hace referencia a llevar adelante una obra o un negocio. Suele ser un proyecto que se desarrolla con esfuerzo y haciendo frente a diversas dificultades, con la resolución de llegar a un determinado punto (Frese, y Rauch, 2000).

Vistas las tres definiciones, no es difícil entender que son tres procesos psicológicos complementarios y diferenciados. La creatividad, es un proceso primario de ideación, que está en la base de los otros dos. La innovación es un proceso secundario, ya aplicado, en donde la creatividad se aplica a la creación de un nuevo proceso, un producto o un servicio. El emprendimiento es un proceso psicológico terciario, que lleva consigo poner en marcha algún tipo de empresa o sistema de explotación de la innovación ya desarrollada. A este conjunto de elementos se le ha denominado cultura emprendedora (Junta de Andalucía, 2011, pág. 119). Ver Figura 1.

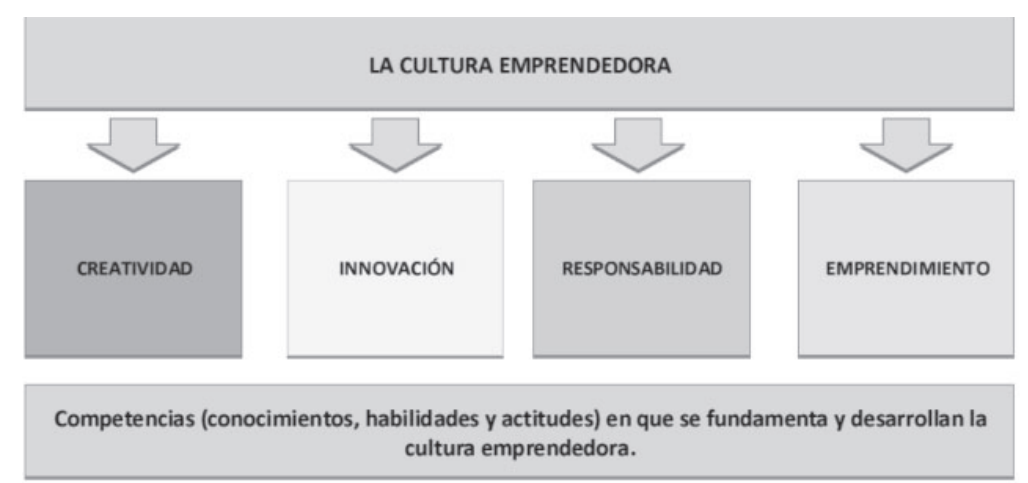

Figura 1. Subprocesos de la cultura emprendedora

Creatividad, Innovación, Emprendimiento: La cadena de valor $I+D+i$

Tal y como se ha planteado, los tres subprocesos son partes secuenciales y recurrentes de la creación, desarrollo e implementación de cualquier creación humana. La creatividad, en esencia, es un proceso psicológico que se sitúa en el ámbito de las ideas, las teorizaciones, o 
las ideaciones para resolver problemas. El acto de crear es, inicialmente, conceptual. Da igual que sea una nueva teoría científica que una relación aplicada entre objetos para resolver un problema dado. Nadie puede negar la creatividad o el descubrimiento de nuevas relaciones de nuestros antepasados, al poner en relación el petróleo con un coche, o una moneda y un carro de un supermercado - a priori, inconexos. Por ello, la creatividad, el descubrimiento y la investigación están íntimamente relacionadas. De hecho, en ciencia, la investigación, entendida como el conocimiento preciso de las relaciones entre conceptos, principios o elementos de la realidad, es imprescindible, para realizar nuevas propuestas o creaciones científicas. En este caso podríamos hablar de creatividad ideacional o científica, propia de la investigación científica. Este tipo de creatividad primaria es esencial para el avance del conocimiento y de la ciencia, pero per se no produce innovación.

Sin embargo, existe otra modalidad de creatividad, más aplicada, que si sitúa en la base, del desarrollo de nuevos procesos, productos y servicios: la innovación o desarrollo. En este ámbito se situarían el desarrollo de dispositivos, cambios en los procesos de evaluación y tratamiento, en los procesos productivos o de gestión de procesos, productos o servicios. Se debe dar la condición de que exista una producción final evaluable, que sea registrable o, mejor, patentable, para protegerla por su valor de aplicabilidad, para dar respuesta a problemas o demandas de la sociedad, en cualquiera de sus actividades económicas, científicas o sociales. Podemos considerar que hay innovación, cuando se producen las ideas que se han materializado en un desarrollo tecnológico de nuevos procesos, productos y servicios que son comercializables o que genera algún tipo de beneficio. Este proceso, puede -aunque no siempre es así- estar muy ligado al de la creación investigadora, pero son independientes, por cuanto el conocimiento preciso de los procesos o problemáticas científicas, de nuevos modelos científicos (conceptos), puede ayudar a poner en marcha nuevos desarrollos innovadores (procedimientos) que supongan la innovación, pero no siempre ocurre así. Se pueden producir cambios en procesos, productos y servicios, en un nivel aplicado, sin que procedan de la teoría o de la ciencia. Sin embargo, en los desarrollos tecnológicos de alto nivel, tales como nuevos sistemas de evaluación, de intervención o tecnológicos aplicados con formato TICs, es imprescindible el conocimiento del arte o del estatus científico del problema, o, también, los desarrollos tecnológicos previos, si se desea realizar ajustes o cambios realmente innovadores. 
Finalmente, existe una creatividad aplicada al campo de la comercialización, denominada emprendimiento, centrada en la explotación o la transferencia de esos productos, procesos o servicios, ya desarrollados, a través de sistemas reglados de transferencia o explotación mercantil, tales como la creación de empresas o transferencia a otras, de tales desarrollos tecnológicos. Esta última actividad, es la que realmente crea riqueza y negocio, habida cuenta, que las dos anteriores, sin este eslabón final de la cadena, no lo producen.

Cuando las tres actividades creativas citadas (creatividad investigadora, desarrollo innovador y transferencia emprendedora) se ponen a trabajar conjuntamente se produce la construcción de la cadena de valor I (Investigación científica) $+D($ Desarrollo tecnológico $)+i$ (transferencia emprendedora), en cualquier campo de conocimiento. Un ejemplo de ello son las propuestas recientes, en el campo de la Psicología (De la Fuente, y Vera, 2010)

Perfiles profesionales y posicionamientos en la cadena de valor $I+D+i$

Sin embargo, los perfiles profesionales generados, en la trayectoria económica, académica y social reciente, no siempre han contribuido a que se produzca una integración de los tres elementos, es decir, la investigación, el desarrollo tecnológico y la transferencia de la innovación. Podemos encontrar diferentes posicionamientos en torno a la cadena de valor I+D+i. Habitualmente, los profesionales de la investigación están situados en este eje de la misma, y, por tanto, más centrados en la producción de ciencia y conocimiento que nos permita conocer mejor los mecanismos explicativos de cada objeto de estudio; por tanto, la actividad cotidiana es crear modelos, teorías, relaciones que describan, expliquen, predigan los aspectos estudiados. En algunas ocasiones, estos profesionales, realizan desarrollos tecnológicos aplicados para evaluar o intervenir en los problemas objetos de estudio.

Los profesionales aplicados, sin embargo, están más interesados en el segundo eslabón de la cadena, en los desarrollos tecnológicos específicos que les permitan gestionar e intervenir en la realidad directa de la profesión, por lo que gran parte de su actividad se centra en identificar problemas y tomar decisiones para evaluarlos e intervenir sobre ellos, dando respuesta a la demanda planteada. En esta práctica profesional pueden surgir nuevos desarrollos profesionales, que pueden implementarse como innovación, en la propia empresa o ámbito de 
trabajo. En este caso la innovación es un componente implícito requerido en la práctica profesional para dar respuesta a las nuevas demandas. Sin embargo, tampoco ocurre siempre así. Este hecho es lo que se ha denominado "ruptura o falta de integración de la cadena de valor I+D+i” (De la Fuente y Vera, 2010), representado gráficamente, en la Figura 2.

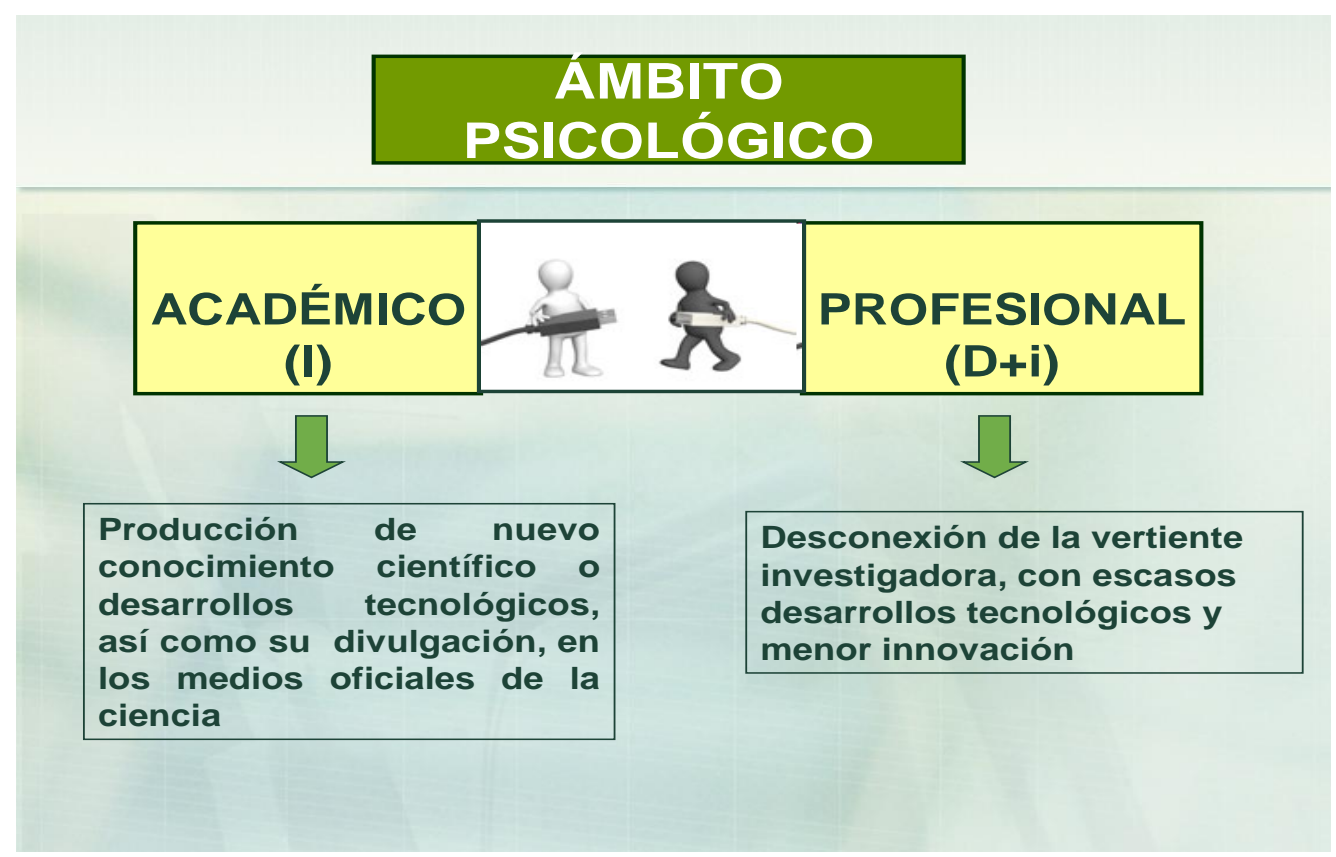

Figura 2. Desconexión entre los elementos de la cadena de valor I+D+i

Las consecuencias de tal concepción han sido:

1) Focalización de cada ámbito en el conocimiento y el ámbito de sus competencias. Es decir, los investigadores se dedican, preferentemente, a la actividad de investigación, con escaso interés por el desarrollo tecnológico y la transferencia de conocimiento a la práctica profesional empresarial. Los profesionales, por su parte, desean dar respuesta a las demandas profesionales e innovar, aunque no siempre existe una conexión con los avances investigadores.

2) Dificultad de definir proyectos comunes e integrados, que respondan a la filosofía de la Investigación + Desarrollo + innovación, que generen nuevos puestos de trabajo y productos novedosos y competitivos en el mercado. 


\section{Por qué la Sociedad del Conocimiento necesita personas con creatividad, innovación y emprendimiento}

Existe numerosa evidencia referida a que los procesos productivos, en el mercado laboral propio de la Sociedad del Conocimiento, requieren personas capaces de acelerar el proceso de innovación, habida cuenta de las características del mercado en la misma (Reyes, 2009): (1) un constante incremento del del conocimiento y de la tecnología, (2) el ciclo de vida de los productos o servicios es cada vez más corto, (3) se requiere mayor velocidad para ingresar en el mercado (time to market), (4) se trabajan con una nueva oferta basada en el valor más que en el costo (value proposition), (5) innovaciones radicales. Por todo ello, la innovación está en el núcleo de la creación de valor, a partir del conocimiento, la creatividad y el emprendimiento. Ver Figura 2 y Anexo I.

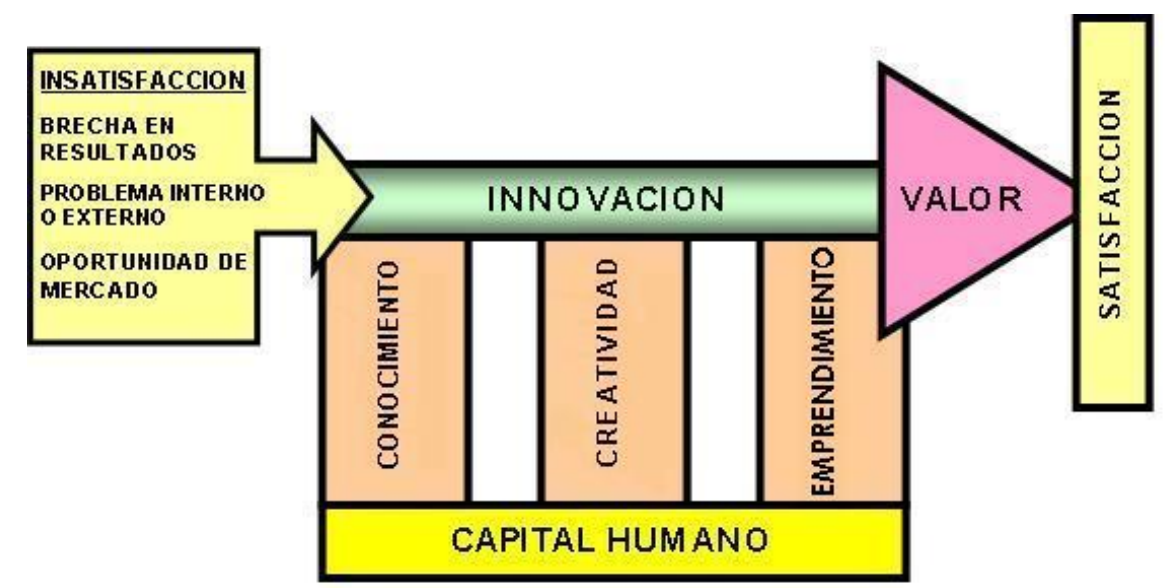

Figura 2. La innovación como eje de la creación de valor (Tomado de Reyes, 2009)

\section{Necesidad y aportaciones de la Psicología de la Innovación y del Emprendimiento}

Como ya se ha fundamentado, creatividad, innovación y emprendimiento, son tres macroprocesos psicológicos en intensa relación e interdependencia, con relaciones pendientes aún de definir. Tradicionalmente, la Psicología de la Creatividad surgió históricamente como disciplina y ámbito de investigación para establecer con rigor y precisión el papel de este proceso, en la persona humana (Csikszentmihalyi, 1998): etiología, características, medidas, evaluación, intervención y mejoras. En estos momentos, es fácil encontrar en el ámbito académico y profesional, categorizaciones, sistemas de evaluación y mejora, referidos a la creatividad, convirtiéndose en una competencia humana de adaptación al medio imprescindible. No 
obstante, este camino está por construir, cuando nos referimos a los procesos de innovación y de emprendimiento. De ahí la necesidad de sentar las bases para su establecimiento como objeto de estudio, a través de la Psicología de la Innovación y del Emprendimiento.

Necesidad de la Psicología de la Innovación y del Emprendimiento como campo disciplinar aplicado

Una revisión rápida en Google Scholar y en las bases de datos científicas más comunes sobre este concepto pone de manifiesto varios aspectos, dignos de análisis:

1. En gran parte de las publicaciones y sitios webs se refieren a la innovación, aplicada al campo empresarial, intentando extrapolar este concepto al ámbito psicológico.

2. En el caso del emprendimiento, dadas sus características empresariales la conexión con dicho ámbito es mayor aún.

3. La mayoría de los trabajos se centran en relaciones o problemáticas discretas relativas al componente psicológico de la innovación y del emprendimiento, y pocas a la visión disciplinar de este objeto de estudio.

Hacia la definición de un estatus científico de la Psicología de la Innovación y del Emprendimiento

El objeto de estudio de este campo disciplinar se puede referir como el análisis de los comportamientos y competencias de Innovación y Emprendimiento, en interacción con el contexto en el que se produce y de los procesos psicológicos implicados, a través de los métodos y técnicas propios de la ciencia psicológica. Frese, y Rauch, (2000) establecen que el estudio del emprendimiento está en la frontera entre la psicología del trabajo, psicología organizacional y la psicología del marketing. Prácticamente todos los aspectos que estudia la psicología están implicados en el estudio de los emprendedores.

Ya existen trabajos que han planteado esta problemática, referida a las relaciones entre creatividad, oportunidades e innovación (Farr, Sin, y Tesluk, 2003; Mumford, 2003; Shane y Venkataraman, 2000), así como el papel de la Psicología del Emprendimiento (Frese, 2009). De manera complementaria, se ha investigado la relación entre la cultura organizacional, el papel del clima y la creatividad (Anderson y West, 1998; Baer, Frese, 2003; Huelsheger, An- 
derson, y Salgado, 2009; Matson, 1996). Las relaciones entre la creatividad y la innovación como oportunidad, también han sido objeto de interés investigador (Baron, y Ensley, 2006; DeTienne y Chandler, 2004; Shane, 2000).

Otras investigaciones se han centrado en el conocimiento de los factores contextuales de la innovación y del emprendimiento (Aldrich y Martinez, 2001; Covin, y Slevin, 1999; Kodithuwakku, y Rosa, 2002), o en el papel de los factores personales de la innovación y el emprendimiento, tales como el optimismo emprendedor y otras características (Baum, Locke, y Smith, 2001; Frese, 2009). Hmieleski, K. M., \& Baron, 2009; Rauch, y Frese, 2007). A nivel de estrategia de innovación y emprendimiento, se ha definido el valor y la funcionalidad de las conductas de organización de los recursos y la planificación de las ventajas competitivas (Terpstra, y Olson, 1993; Rauch, Wiklund, Lumpkin, y Frese, 2009; Winborg, y Landstrom, 2000), la importancia de la ejecución del plan de negocio, la implementación, la experimentación de soluciones de negocio y la competencia de improvisación (Baker, y Miner y Eesley, 2003; Sarasvathy, 2001; Frese, Krauss, Keith, Escher, Grabarkiewicz, Luneng, et al., 2007), las características definitorias de liderazgo emprendedor y su capacidad transformadora de la realidad (Baum, Locke y Kirkpatrick, 1998; Ensley, Pearce, y Hmieleski, 2006 Ling, Simsek, Lubatkin, y Veiga, 2008) o su capacidad de trabajo en situaciones de ambigüedad o la hipótesis del ambidestrismo en situaciones corporativas (Bledow, Frese, Anderson, Erez, y Farr, 2009). Incluso, se ha investigado el papel del contexto familiar en el emprendimiento (Wadwa, Aggarwal, Holly y Salkever, 2009).

Adhmad (2007) presenta un estudio en el que examinó la relación entre las competencias empresariales y el éxito empresarial en el contexto de las PYME en Australia y Malasia. Se adoptó un enfoque de "métodos mixtos" llevándose a cabo dos estudios de manera secuencial (Estudio 1 y Estudio 2). En el Estudio 1, un método cualitativo fue adoptado en el que se realizaron entrevistas individuales con 20 empresarios de Australia - 10 y 10 de Malasia - que operaban las PYMES en los sectores manufacturero y de servicios. El objetivo era conocer las conductas que delimitan las competencias, y por lo tanto permitir la identificación de competencias emprendedoras que son específicas del contexto y de relevancia en el entorno empresarial actual. Un análisis de contenido de los datos de las entrevistas, ha identificado 12 ámbitos de competencia: Estratégicos, Compromiso, Conceptual, Oportunidad, Organización y la principal personal, Relación, y Aprendizaje, Técnica, Ética, Responsabilidad social, y Familiariedad. Es importante destacar que nueve de estas categorías están bien representa- 
das en los modelos existentes de competencias empresariales, proporcionando evidencia de que estos modelos ofrecen un razonable grado de generalización transcultural. Sin embargo, surgieron tres nuevas categorías, a saber, la responsabilidad ética, social y familiariedad (en sentido amplio que ver con el papel de la "familia" en el éxito de la construcción de negocios). Hubo pruebas de competencias responsabilidad ética y social, tanto en los datos de Australia y Malasia, mientras que la familiariedad era específica para los datos de Malasia y bien puede reflejar la orientación colectivista de la cultura malaya. La identificación de estas categorías adicionales de competencias empresariales sugiere que los modelos existentes pueden necesitar ser revisados para mejorar aún más su aplicabilidad a la medición de competencias empresariales en los distintos contextos culturales.

En el Estudio 2 (Parte 1), se procedió a la validación del modelo de competencias emprendedoras. Se trataba de determinar el rigor psicométrico del modelo y establecer las propiedades psicométricas de todas las variables dependientes (medidas de éxito en los negocios) y covariables (es decir, el entorno empresarial y las orientaciones de los emprendedores culturales) utilizando una muestra de 391 empresarios de las PYMES (179 australianos y 212 malasios). Aparecieron dos modelos de ajuste de competencias empresariales - el "Integral" y el modelo "parsimonioso". El modelo integral reveló que las doce áreas de competencias fueron percibidas como relevantes para los resultados de los empresarios de PYME en Australia y Malasia, a pesar de que los comportamientos que definen la organización y las competencias de dominio principal, así como el dominio de las competencias familismo (más tarde llamado "Apoyo y Cooperación" para Australia debido a la omisión de elementos relacionados con la" familia ") eran algo diferentes. Las otras 10 áreas de competencia identificadas en el modelo integral parecía ser invariantes en todos los países. Por otro lado, el modelo parsimonioso de competencias empresariales mostraron que, si bien algunas áreas de competencia son universales en la naturaleza (es decir, la Opportunidad, Conceptual, Aprendizaje y Ética), otras estaban en un país específico (es decir, Relación, Responsabilidad Social, y Familiariedad). Se argumentó que las diferencias podrían deberse a variaciones culturales entre los países, en particular, el grado de individualismo versus colectivismo. El Estudio 2, Parte 2, probó la vía causal entre las competencias empresariales y el éxito empresarial y la posible influencia de las covariables usando un modelo de ecuaciones estructurales (SEM) como procedimiento. Los resultados mostraron que las competencias empresariales eran fuertes predictoras de éxito empresarial en las PYME, para Australia y Malasia. También se encontró que los entornos empresariales, tanto benignos y estables se relacionaron significativamente con el éxito del 
negocio en Australia, mientras que sólo un entorno estable se asoció significativamente con el éxito en Malasia. Por otra parte, las variables ambientales mostraron un camino menos fuerte para el éxito de las competencias. Cuando el modelo parsimonioso de competencias empresariales se utilizó en la estimación del modelo, la asociación entre las competencias empresariales y el éxito empresarial fue más fuertemente evidente en ambientes hostiles y dinámicos que en los ambientes más benignos y estables (tanto para Australia y Malasia). Los resultados también mostraron que las orientaciones culturales (tanto colectivismo como tolerancia a la ambigüedad) tienen efectos positivos sobre competencias emprendedoras en Malasia, pero no en Australia (es decir, el individualismo y la tolerancia a la ambigüedad). El efecto de la educación en competencias empresariales era mixto, significativo sólo para el modelo integral de Australia, pero significativo para los modelos, a la vez completos y parsimoniosos, de Malasia. Los efectos de la formación antes y después de la puesta en marcha, así como la experiencia laboral previa en competencias empresariales parecían ser no significativas en ambos contextos. Sobre la base de los resultados de la tesis, se concluyó que la percepción subjetiva de las competencias empresariales son predictivas de auto-informes de éxito de las PYME, tanto en Malasia como Australia. Los modelos que describen el éxito en ambos países son consistentes con los modelos existentes de competencia empresarial a pesar de que las diferencias en los comportamientos que definen un dominio se evidencian entre los países como son los dominios que definen con éxito un modelo parsimonioso en cada país. Estos resultados se interpretan como apoyo a un programa de formación que identifica las capacidades empresariales como un importante pre-requisito para el éxito empresarial de las PYME.

\section{Las sinergias entre la innovación y el emprendimiento}

Zhao (2005) ha informado de una investigación para contribuir a la comprensión de la naturaleza complementaria de la iniciativa empresarial y la innovación a través de un estudio empírico de diversas organizaciones, y para desarrollar un marco integrador de la interacción entre el espíritu empresarial y la innovación. El estudio tiene un enfoque cualitativo para explorar las sinergias entre la iniciativa empresarial y la innovación y en el análisis de los factores que fomentan la interacción entre los dos. Se realizaron estudios de caso de seis organizaciones empresariales e innovadoras y entrevistas en profundidad con los altos directivos. Se llevó a cabo una revisión exhaustiva de la literatura de la iniciativa empresarial y la innovación. El estudio encontró que: (1) el espíritu empresarial y la innovación están positivamente relacionados entre sí e interactúan para ayudar a una organización a prosperar, ya que la ini- 
ciativa empresarial y la innovación son complementarios, y (2) una combinación de los dos es vital para el éxito organizacional y la sostenibilidad, en este mundo tan dinámico y cambiante. La iniciativa empresarial y la innovación no se limitan a las etapas iniciales de una nueva empresa, sino que son procesos dinámicos e integral en las organizaciones empresariales e innovadoras, mientras que la cultura organizacional y el estilo de gestión son factores cruciales que afectan el desarrollo del comportamiento emprendedor y la innovación en las organizaciones. Esta investigación muestra, por tanto, que el espíritu empresarial y la innovación deben ser considerados como una práctica continua y cotidiana en las organizaciones

\section{Un nuevo programa educativo emergente: Educación para la Competencia en Innova- ción y Emprendimiento}

\section{Competencia en innovación y emprendimiento}

La competencia en innovación y emprendimiento ya es imprescindible, en la Sociedad del Conocimiento del siglo XXI que nos ha tocado vivir, especialmente, con la actual crisis económica, y con el devenir de los grandes cambios que se producen en los modelos de profesión y de negocio. Por ello, en base a las aportaciones de las investigación psicológica sobre innovación y emprendimiento, se pueden establecer algunas características comportamentales de la competencia para la innovación y el emprendimiento, tal y como se resume en la Tabla 1.

Tabla 1. Características comportamentales de la competencia para la innovación y el emprendimiento

\begin{tabular}{lll}
\hline & Competencia en Innovación & Competencia en emprendimiento \\
\hline Personales & necesidad de cambio & auto-eficacia en el cambio \\
autorregulación & resiliencia, sobreponerse al fracaso \\
apertura a la experiencia & optimismo empresarial \\
& gestion emocional & gestión de la presión \\
& control del éxito \\
Sociales & habilidades sociales & habilidades de gestión de grupos \\
Cognitivo & & pensamiento bajo incertidumbre
\end{tabular}


generación de proyectos

creatividad innovadora

Lingüístico comunicación adecuada

persuasión aplicación de proyectos a la empresa

creatividad para la transferencia

gestión de la información

transmisión de misión y visión

Aprendizajes curriculares propios de la innovación y del emprendimiento

Educar para la competencia en innovación y emprendimiento supone diseñar y desarrollar procesos de enseñanza-aprendizaje -tanto en los contextos formales, como no formales e informales-, que contribuyan a la construcción, incorporación e integración de los comportamientos propios de saber, poder y querer innovar y emprender, en las nuevas generaciones del siglo XXI. Tal planteamiento debe basarse en una agenda de aprendizaje para la innovación y el emprendimiento, que puede ser considerado, un aprendizaje curricular transversal o específico. Existen diferentes propuestas incipientes hasta el momento (ver Anexo II), e incluso algunas legislaciones han establecido elementos sustantivos de un programa educativo para la innovación y el emprendimiento (Junta de Andalucía, 2011). A continuación, se proponen algunos aprendizajes propios de esta competencia, en la Tabla 2:

Tabla 2. Aprendizajes propios de la competencia para la innovación y el emprendimiento

\begin{tabular}{|c|c|c|c|}
\hline \multirow[t]{7}{*}{ Saber } & Hechos & - Conocimiento de la Sociedad del & - Tipos de negocios y empresas \\
\hline & & Conocimiento. & . Requerimientos y funcionamiento \\
\hline & & - Relaciones entre ciencia, tecnología y & · Legislación. \\
\hline & & sociedad. & · Economía. \\
\hline & & - Modelo económico basado en TICs & - Modelo de negocio basado en TICs \\
\hline & Conceptos & - Concepto de innovación. & - Concepto de emprendimiento. \\
\hline & & - Invenciones, registros y patentes. & · Empresas de Base Tecnológica, Biotec- \\
\hline
\end{tabular}

Principios · Principios de la innovación · Principios del emprendimiento 


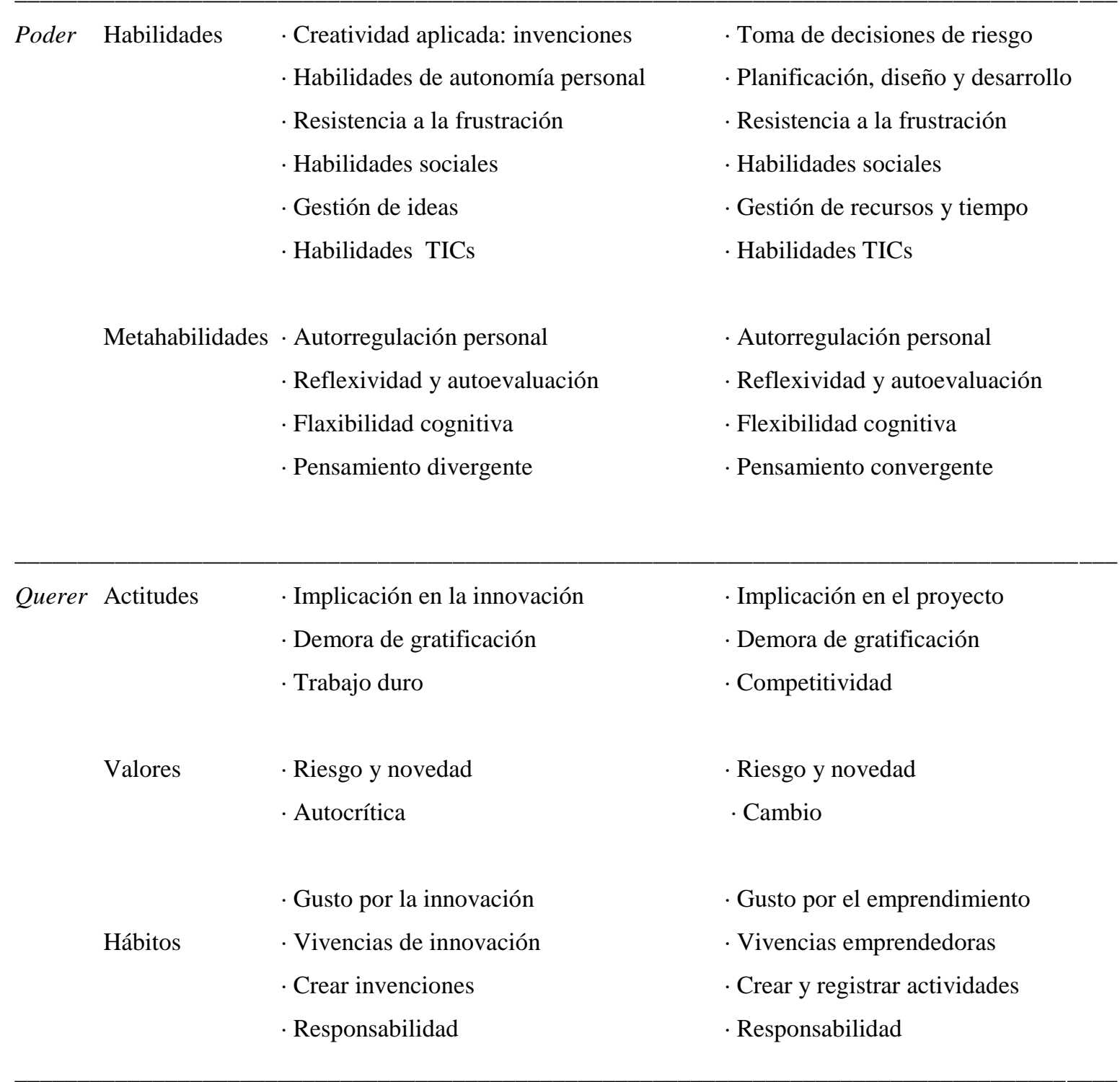

Un modelo educativo basado en nuevos contenidos, nuevas formas de aprender, creativas, innovadoras y emprendedoras

En este nuevo modelo o programa educativo emergente, los profesores no son un elemento secundario más, sino que deben configurarse como los verdaderos protagonistas y líderes del cambio, junto al alumnado. El plan deberá propiciar el posicionamiento del capital social más relevante del sistema educativo, la masa crítica del personal docente, como la palanca de cambio que permitirá que esta transformación se produzca. El profesorado, en este nuevo panorama, deberá ser el gran facilitador para que la cultura emprendedora, la innova- 
ción y la creatividad se conviertan en los pilares de la educación de los niños, jóvenes, universitarios y adultos.

Por ello, deberán establecerse los recursos y los dispositivos específicos de apoyo al profesorado, para primar el talento, la excelencia emprendedora y la creatividad de los propios cuerpos de docentes. De esta forma, en este nuevo panorama educativo los profesores y profesoras pasan a convertirse en el recurso crítico más valioso de nuestra sociedad, pues deben ser los actores que deben protagonizar el cambio.

Igualmente crítica será la transformación, no sólo de los contenidos educativos, sino de las propias metodologías de enseñanza, que deberán evolucionar para ser capaces de transmitir los nuevos valores de la iniciativa, la innovación y la creatividad. Para ello, la única vía posible es el desarrollo e implantación de metodologías de enseñanza innovadoras y creativas. No es posible transmitir nuevos conocimientos, nuevas habilidades y nuevos valores manejando unas metodologías de enseñanza pasivas y unidireccionales.

\section{Conclusiones y apuestas de futuro}

El espíritu emprendedor se refiere a una competencia que se genera al interior de cada persona que, por decisión libre y personal, persevera en la satisfacción de sus motivaciones, traspasando su estabilidad para lograr un mejor desarrollo de sí mismo, de los otros y del entorno, con pasión, riesgo y sacrificio. Esta actitud se presenta en aquellas personas que tienen el deseo de conocer sus propias motivaciones, saber quién soy, qué quiero y para dónde voy, y que -con una fuerte convicción por el logro-, imaginan, buscan y generan nuevas oportunidades para satisfacer sus motivaciones. Presentan iniciativa para adelantarse a los cambios y a los demás, con visión de futuro y agregación de valor, planteando ideas creativas e innovadoras, siendo capaz de influenciar e integrar a otros en su propia idea, como integrarse a ideas desarrolladas por otras personas. Planifican y organizan los recursos y medios para llevar a cabo sus iniciativas, analizando el entorno y asumiendo los riesgos y consecuencias que una idea innovadora conlleva. Es una persona que tiene la facilidad de enfrentar diversas situaciones de manera adecuada, pues dispone de una elevada estabilidad y fortaleza emocional, lo que le permite adaptarse rápido y enfrentar los problemas como oportunidades de mejora, replanteándose y perseverando hasta conseguir sus objetivos, actuando siempre en un plano 
moral y de valores que respeta la vida, la libertad, mejorando las condiciones de vida de la comunidad y el sentido de trascendencia.

El programa futuro de esta línea de trabajo debe tener como objetivo acercar el emprendimiento como un proceso entendido como una actitud ante la vida, más allá de la formación de empresas, constituyéndose en un elemento central para el fortalecimiento de equipos de trabajo, mejoramiento de procesos, y un elemento diferenciador en la formación escolar y universitaria. Sin embargo, para incorporar estos elementos de forma sostenida a la escuela, universidad, empresa, instituciones en general e incluso en la propia familia, se requieren personas y profesionales con las competencias necesarias para comprender, integrar y contextualizar el emprendimiento y la innovación a la realidad de sus instituciones, fortaleciendo la calidad y eficiencia en el logro de sus objetivos. Ver anexo II.

En el nivel no universitario, es imprescindible sentar las bases competenciales mediante programas de intervención primaria. Un papel protagonista en los centros educativos, podrían ser los Departamentos de $\mathrm{I}+\mathrm{D}+\mathrm{i}$, encargados de promover, dinamizar e implementar dichas competencias (De la Fuente, 2012; De la Fuente y Zapata, 2012). En el nivel universitario, ya es una realidad, en muchas universidades anglosajonas, la integración de un currículum transversal de competencias para la innovación y el emprendimiento. También en el ámbito español universitario se ha realizado un esfuerzo por dinamizar e implementar el espíritu emprendedor, a través de la creación de Empresas de Base Tecnológica (http://cms.ual.es/UAL/investigacion/ebts/index.htm). Desgraciadamente, ésta es una realidad más evidente en las áreas científico-tecnológicas clásicas por excelencia, tales como las áreas de industria, bio-ciencia, aeroespacial, ambiental, software, servicios de manufacturación (Wadwa, Freeman, y Rissing, 2008; Wadhwa, Rissing, Saxenian, y Gereffi, 2007; Wadhwa, V., Rissing, Saxenian, y Gereffi, 2007).

Por ello, la Psicología y la Educación, no deberían renunciar a tener el protagonismo que merecen, en este proceso de construcción personal y social de los profesionales emprendedores del siglo XXI. Es por tanto, una oportunidad inigualable para aunar tradición (Psicología de la Creatividad) e innovación (Psicología de la Innovación y del Emprendimiento) en la Psicología contemporánea (Carpintero, 2001). No debemos perderla, como ciencia y como profesión. 


\section{Agradecimientos}

Este trabajo se ha realizado en el marco del Proyecto I+D Ref. EDU2011-24805 (2012-2014)

Madrid: Ministerio de Ciencia e Innovación y Fondos DEDER.

\section{Referencias}

Ahmad, N. H.A (2007). A cross cultural study of entrepreneurial competencies and entrepreneurial success in SMEs in Australia and Malaysia. Tesis Doctoral. University of Adelaide, Adelaide Graduate School of Business, Australia.

Aldrich, H. E., y Martinez, M. A. (2001). Many are called, but few are chosen: An evoluationary perspective for the study of entrepreneurship. Entrepreneurship: Theory \& Practice, 25(4), 41-56.

Anderson, N. R., y West, M. A. (1998). Measuring climate for work group innovation: Development and validation of the team climate inventory. Journal of Organizational Behavior, 19, 235-258.

Baer, M., y Frese, M. (2003). Innovation is not enough: Climates for initiative and psychological safety, process innovations, and firm performance. Journal of Organizational Behavior, 24, 45-68.

Baker, T., Miner, A., y Eesley, D. (2003). Improvising firms: Bricolage, account giving, and improvisational competency in the founding process. Research Policy, 32, 255-276.

Baron, R. A., y Ensley, M. D. (2006). Opportunity recognition as the detection of meaningful patterns: Evidence from comparisons of novice and experienced entrepreneurs. Management Science, 52, 1331-1344.

Baum, J. R., Locke, E. A., y Kirkpatrick, S. A. (1998). A longitudinal study of vision and vision communication to venture growth in entrepreneurial firms. Journal of Applied Psychology, 83, 43-54.

Baum, J. R., Locke, E. A., y Smith, K. G. (2001). A multi-dimensional model of venture growth. Academy of Management Journal, 44, 292-303.

Bledow, R., Frese, M., Anderson, N. R., Erez, M., y Farr, J. L. (2009). A dialectical perspective on innovation: Conflicting demands, multiple pathways, and ambidexterity. Industrial and Organizational Psychology: Perspectives on Science and Practice, 2(3), 305337.

Carpintero, H. (2001). Tradición e innovación en la Psicología Contemporánea. Papeles del Psicólogo, 78, 3-10 
Covin, J. G., y Slevin, D. P. (1999). Pioneers and followers: Competitive tactics, environment, and firm growth. Journal of Business Venturing, 15, 175.

Csikszentmihalyi, M. (1998) Creatividad. El fluir y la psicología del descubrimiento y la invención. Barcelona: Paidós psicología.

De la Fuente, J. (2012). Desde la orientación profesional hasta las últimas propuestas de $l+D+i$ en Psicología y educación. Jornadas Profesionales del Instituto EOS. Madrid: EOS. http://www.eos.es/Noticias.html

De la Fuente, J. y Vera, M. M. (2010). Educational Psychology and R \& D \& i: a strategic action programme for the twenty-first century. Papeles del Psicólogo, 31 (2), 161-170

De la Fuente, J. y Zapata, L. (2012). Creación de un Departamento $I+D+i$ en el ámbito Psicoeducativo. Almería (España): Education \& Psychology I+D+i, e-Publising Series $\mathrm{I}+\mathrm{D}+\mathrm{i}$.

DeTienne, D. R., y Chandler, G. N. (2004). Opportunity identification and its role in the entrepreneurial classroom: A pedagogical approach and empirical test. Academy of Management Learning and Education, 3, 242-257.

Ensley, M. D., Pearce, C. L., y Hmieleski, K. M. (2006). The moderating effect of environmental dynamism on the relationship between entrepreneur leadership behavior and new venture performance. Journal of Business Venturing, 21, 243-263.

Farr, J. L., Sin, H.-P., y Tesluk, P. E. (2003). Knowledge management processes and work group innovation. In L. V. Shavinina (Ed.), International handbook of innovation (pp. 574-586). Elsevier.

Frese, M. (2009). Towards a psychology of entrepreneurship: An action theory perspective. Foundations and Trends in Entrepreneurship, 5, 435-494.

Frese, M., Krauss, S., Keith, N., Escher, S., Grabarkiewicz, R., Luneng, S. T., et al. (2007). Business Owners' Action Planning and Its Relationship to Business Success in Three African Countries. Journal of Applied Psychology, 92, 1481-1498.

Frese, M., y Rauch, A. (2000). Psychology of Entrepreneurship. Entrepreneurship, 4552$4556 ; 2000$

Huelsheger, U. R., Anderson, N. R., y Salgado, J. F. (2009). Team-level predictors of innovation at work: A comprehensive meta-analysis spanning three decades of research. Journal of Applied Psychology, 94, 1128-1145.

Hmieleski, K. M. y Baron, R. A. (2009). Entrepreneurs' optimism and new venture performance: A social cognitive perspective. Academy of Management Journal, 52, 473-488. 
Junta de Andalucía (2011). Decreto 219/2011, de 28 de junio, por el que se aprueba el Plan de Fomento de la de la Cultura Emprendedora en el Sistema Educativo Público de Andalucía (BOJA no 137, de 14 de Julio). Sevilla: JA, Consejería de la Presidencia.

Kodithuwakku, S. S., y Rosa, P. (2002). The entrepreneurial process and economic success in a constrained environment. Journal of Business Venturing, 17, 431-465.

Ling, Z., Simsek, Z., Lubatkin, M. H., y Veiga, J. F. (2008). Transformational leadership's role in promoting corporate entrepreneurship: Examining the CEO-TMT interface. Academy of Management Journal, 51, 557-576.

Mumford, M. D. (2003). Where have we been, where are we going? Taking stock in creativity research. Creativity Research Journal, 15, 107 - 120.

Matson, J. V. (1996). Innovate or die: Personal perspective on the art of innovation. Monroe, Wisconsin: Paradigm Press Ltd.

Rauch, A. y Frese, M. (2007). Let's put the person back into entrepreneurship research: A meta-analysis on the relationship between business owners' personality traits, business creation and success. European Journal of Work and Organizational Psychology, 16, 353-385.

Rauch, A., Wiklund, J., Lumpkin, G. T., y Frese, M. (2009). Entrepreneurial orientation and business performance: Cumulative empirical evidence. Entrepreneurship Theory and Practice, 33, 761-787.

Reyes, P. (2009). El rol de la Psicología en la Innovación. Documento recuperado el 05/08/12: http://blog.pucp.edu.pe/media/avatar/530.pdf

Roth, E. y Lacoa, D. (2012). Análisis Psicológico del Emprendimiento en Estudiantes Universitarios: Medición, Relaciones y Predicción. Consultado el 20/09/12: http://ucbconocimiento.ucbcba.edu.bo/index.php/raj/article/viewFile/258/256

Shane, S. (2000). Prior knowledge and the discovery of entrepreneurial opportunities. Organization Science, 11, 448-469.

Sarasvathy, S. D. (2001). Causation and effectuation: Toward a theoretical shift from economic inevitability to entrepreneurial contingency. Academy of Management Review, 26, 243-263.

Shane, S., y Venkataraman, S. (2000). The promise of entrepreneurship as a field of research. Academy of Management Review, 25, 217-226.

Terpstra, D. E., y Olson, P. D. (1993). Entrepreneurial start-up and growth: A classification of problems. Entrepreneurship: Theory \& Practice, 17(3), 5-19. 
Wadwa, V., Aggarwal, R., Holly, K. y Salkever, A. (2009). The anatomy of an Entrepreneur. Family Background and Motivation. Kansas (USA) Ewing Marion Kauffman Foundation. www.kauffman.org

Wadwa, V., Freeman, R. y Rissing, B. (2008). Education and Tech Entrepreneurship. Kansas (USA) Ewing Marion Kauffman Foundation. www.kauffman.org

Wadhwa, V., Rissing, B., Saxenian, A. y Gereffi, G. (2007). Education, Entrepreneurship and Immigration: America's New Immigrant Entrepreneurs, Part II. The Ewing Marion Kaufman Foundation. June 2007. Available online at http://www.soc.duke.edu/globalengineering/papers_educationentrepreneurship.html

Wadhwa, V., Saxenian, A., Rissing, B. y Gereffi, G. (2007). America's New Immigrant Entrepreneurs: Part I. The Ewing Marion Kaufman Foundation. January 2007. Available online at http://www.soc.duke.edu/globalengineering/papers_newimmigrant.html.

Winborg, J., y Landstrom, H. (2000). Financial bootstrapping in small businesses: Examining small business managers' resource acquisition behaviors. Journal of Business Venturing, 16, 235-254.

Zhao, F. (2005). Exploring the synergy between entrepreneurship and innovation. International Journal of Entrepreneurial Behaviour \& Research, 11(1), 25-41. 
Anexo I. ¿Cómo es que nadie pensó en esto antes?
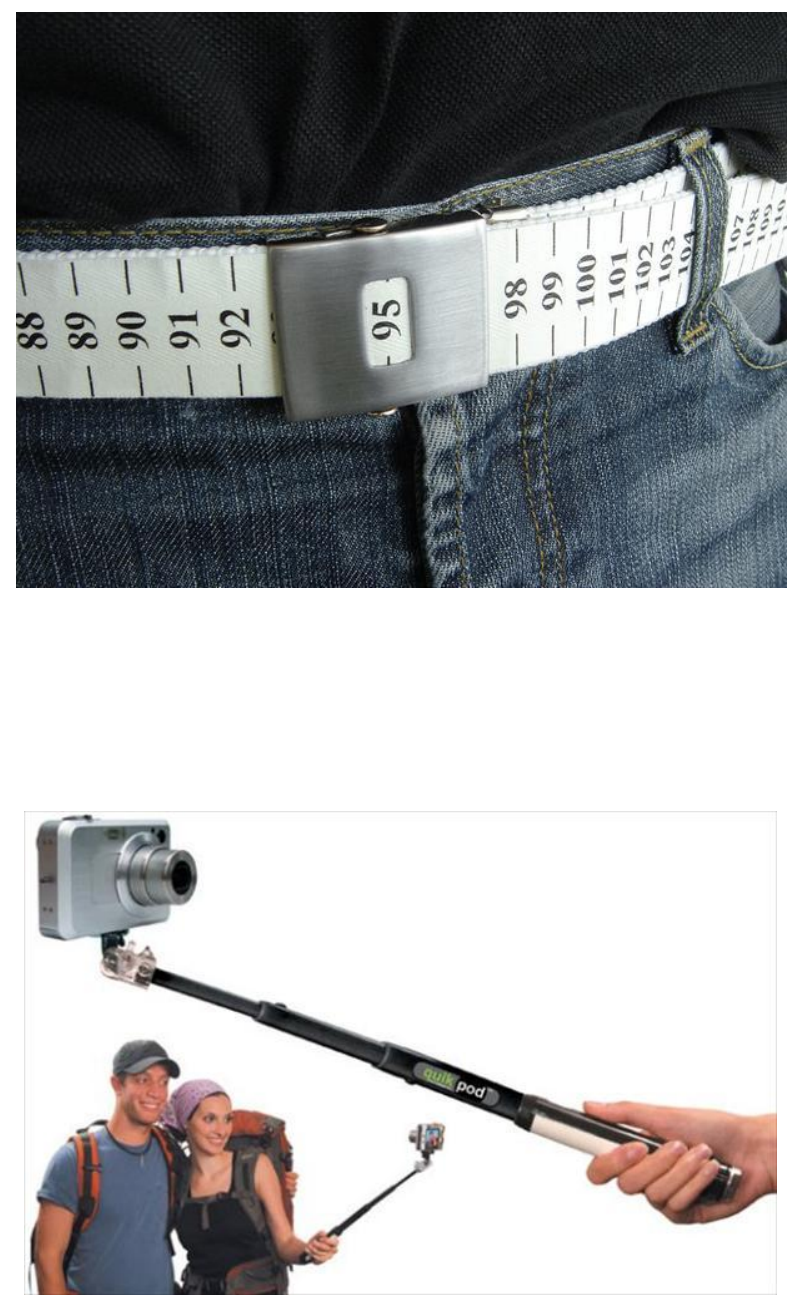

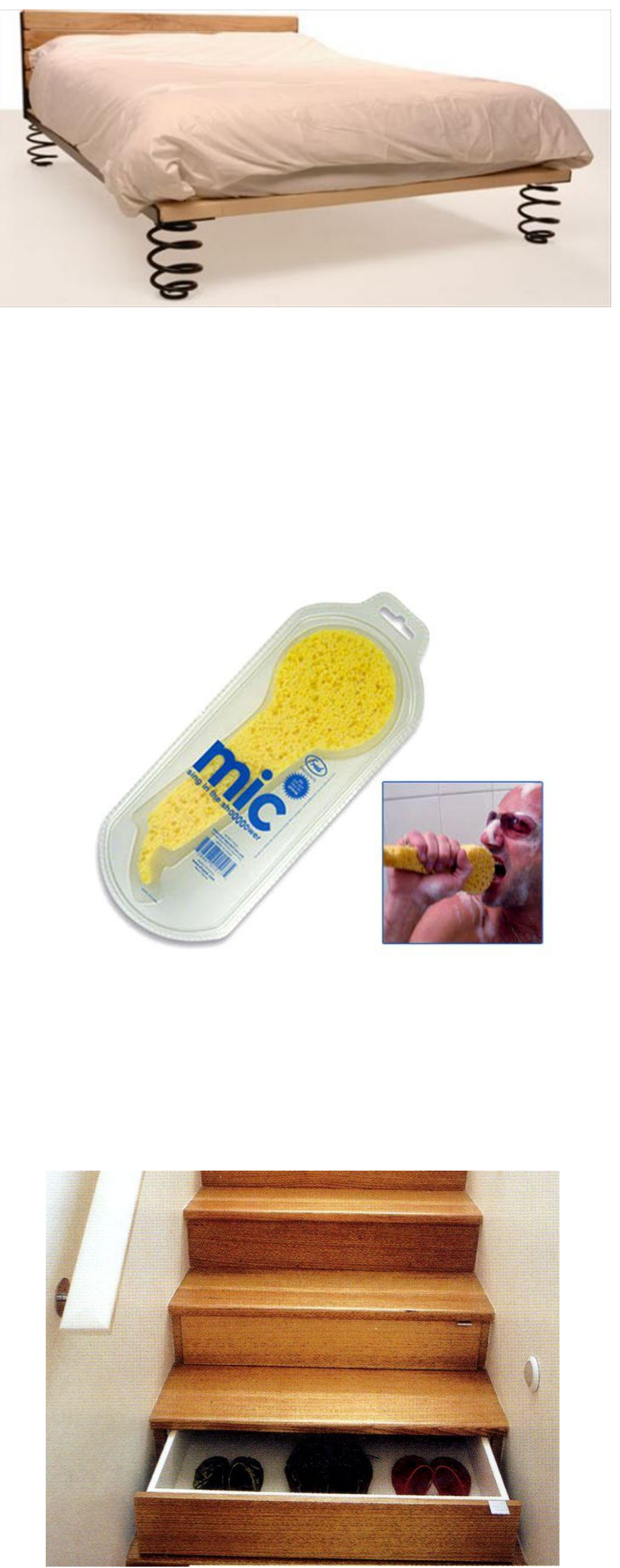
Anexo II. Para saber más:

http://www.cipi2013.com/

http://ec.europa.eu/research/innovation-union/index_en.cfm?pg=videos

http://alfpa.upeu.edu.pe/creatividad/creatividad.htm

http://www.europeanprojects.org/awards

http://fundacioncreate.org/

http://www.education-psychology.com

http://www.juntadeandalucia.es/averroes/ emprender/index.php?option=com $\operatorname{content\& task=view\& id=43\& Itemi}$ $\underline{\mathrm{d}=63}$ 